\title{
Die unendliche Geschichte der informellen Beschäftigung in deutschen Privathaushalten?
}

\author{
Dr. Christa Larsen \\ Geschäftsführerin des Instituts für Wirtschaft, Arbeit und Kultur \\ (IWAK) an der Goethe-Universität, Frankfurt am Main
}

\author{
Sigrid Rand \\ Mitarbeiterin am Institut für Wirtschaft, Arbeit und Kultur \\ (IWAK), Frankfurt am Main
}

Der Privathaushalt als Beschäftigungsort unterscheidet sich wesentlich von Arbeitsplätzen in Firmen und Betrieben, Krankenhäusern oder Verwaltungen. Denn die in Deutschland weitreichenden arbeitsrechtlichen Rahmenbedingungen werden in Privathaushalten bisher nur unzureichend angewandt. Dies liegt zum einen daran, dass es sich beim Haushalt um die Privatsphäre von Menschen handelt, die dem Zugriff öffentlicher Kontrolle weitestgehend entzogen ist. Zum anderen arbeiten in Deutschland die meisten Haushaltshilfen schwarz oder als Illegale, so dass auch hier die arbeitsrechtlichen Voraussetzungen nicht eingefordert werden können oder überprüfbar sind. Im folgenden Artikel möchten wir zunächst darlegen, wie sich die derzeitige Beschäftigungssituation in deutschen Privathaushalten darstellt und aus welchen Gründen in hohem Maße informelle und zum Teil prekäre Beschäftigungsverhältnisse vorliegen. Dass diese Situation nicht per se durch den Arbeitsort Privathaushalt bedingt ist, zeigen wir danach am Beispiel von Frankreich. Dort ist es gelungen, mit gezielten Strategien Haushalte in großem Stile für die sozialversicherungspflichtige Beschäftigung von Haushaltshilfen zu gewinnen. Vor dem Hintergrund dieser erfolgreichen französischen Strategie fragen wir, wie auch in Deutschland die formelle Beschäftigung von Haushaltshilfen systematisch gefördert werden kann.

\section{Wie sieht die Beschäftigung von Haushaltshilfen in deut- schen Privathaushalten aus?}

Immer mehr Haushalte brauchen Haushaltshilfen, weil nur mit ihrer Unterstützung familiäre und berufliche Verpflichtungen erfolgreich vereinbart werden können. Konkret bedeutet dies für viele Frauen, die auch bei voller Berufstätigkeit nach wie vor in der Hauptverantwortung für Familie und Haushalt stehen, dass sie eine verlässliche Betreuung für ihre Kinder organisieren und eventuell eine Reinigungskraft finden müssen. Inzwischen kommt zudem immer mehr in den Blick, dass auch die Versorgung und Pflege von Eltern und Schwiegereltern für erwerbstätige Frauen zu einer Belastungsprobe wird, die sie nicht alleine meistern können. Sie brauchen stundenweise, aber oft auch rund um die Uhr, Haushaltshilfen, die den Verbleib ihrer älteren Angehörigen in den eigenen vier Wänden ermöglichen, ohne dass sie als Töchter, Schwiegertöchter oder Ehefrauen ihre eigene Erwerbsarbeit aufgeben müssten.
Derzeit sind in Deutschland weit über eine Million Haushaltshilfen beschäftigt. Bei den meisten handelt es sich um Frauen, die über 40 Jahre alt sind, einen Migrationshintergrund haben und über keine oder nur eine Qualifikation auf niedrigem Niveau verfügen. Nach Schätzungen des Bundesministeriums für Familie, Senioren, Frauen und Jugend erfolgt die Ausführung dieser sogenannten familienunterstützenden Dienstleistungen in 70 bis 90 Prozent aller Fälle in Form von Schwarzarbeit oder illegaler Beschäftigung, wenn die Haushaltshilfen keinen legalen Aufenthalt in Deutschland haben. ${ }^{1}$ Allein in den Haushalten älterer Menschen sind in Deutschland nach Schätzungen einschlägiger Forschungen ca. 600.000 Frauen illegal beschäftigt. ${ }^{2}$ Die meisten von ihnen kommen derzeit aus Polen und der Slowakei, einige sogar aus Drittstaaten wie Weißrussland oder der Ukraine. Illegal beschäftigte Frauen finden sich vermehrt in den Haushalten Älterer, da insbesondere multimorbide Menschen und/oder dementiell Erkrankte auf zeitlich umfangreiche Betreuungs- und Versorgungszeiten angewiesen sind. Dies impliziert oft auch, dass Haushaltshilfen nicht nur bei älteren Menschen im Haushalt arbeiten, sondern auch dort leben. Fallen demgegenüber in Haushalten Arbeiten an, die mit geringem Zeitaufwand erledigt werden können, wie beispielsweise wenige Stunden Reinigen pro Woche, Kinderbetreuung oder die Erledigung von Hol- und Bringdiensten, dann werden diese Arbeiten vorwiegend in Schwarzarbeit erbracht. Als Schwarzarbeiterinnen sind meistens Frauen aus der Nachbarschaft oder dem Bekanntenkreis tätig.

Die Nachfrage nach Haushaltshilfen nimmt stetig zu, bedingt durch eine immer älter werdende Bevölkerung, die möglichst lange in den eigenen vier Wänden leben möchte und soll, eine wachsende Zahl alleinstehender Menschen, die keine Angehörigen hat, die sie versorgen könnten, sowie durch die Zunahme der Erwerbstätigkeit von Frauen. Nach Schätzungen des französischen Arbeitgeberverbands für Haushaltsangestellte (FEPEM) werden bis zum Jahr 2020 ca. 20 Millionen Personen in Haushalten europaweit beschäftigt sein. Dies zeigt, dass es sich hier um ein bedeutendes Beschäftigungsfeld handelt, das allerdings hochgradig informalisiert ist. Die nicht deklarierte oder informelle Beschäftigung von Haushaltshilfen hat Konsequenzen für alle Beteiligten. Die Haushalte selbst

1 Mackroth, Petra (BMFSFJ), Challenges to family policy in the context of demographic change, Vortrag auf der Konferenz von FEPEM (Fédération des Particuliers Employeurs de France) „How to transform the demographic challenge into an opportunity for quality jobs in Europe" am 8.2.2011 in Brüssel.

2 EU-Projekt: Arbeitsmarktpolitische Maßnahmen zur Reduzierung von illegaler Beschäftigung und Schwarzarbeit in Haushalten älterer Menschen; erhältlich im Internet <www.iwak-frankfurt.de/domesticservices.htm> (Zugriff: 8.4.2011). Larsen, Christa/Rand, Sigrid, Frankreich macht es vor. Strategien zur Qualifizierung und legalen Beschäftigung von Haushaltshilfen, in: Forschung Frankfurt, 2.2010. 
machen sich strafbar und haben keine Garantie in Bezug auf die Qualität und die Zuverlässigkeit der Dienstleistung, auch dann nicht, wenn sich die Dienstleistungen auf schutzbedürftige Personen wie Kinder beziehen. Umgekehrt haben die Haushaltshilfen keinen Anspruch auf Qualifizierung und auch keine formalen Nachweise ihrer Berufserfahrung in diesem Segment, was ihnen den „Sprung“ in andere Branchen erleichtern könnte. Der Mangel an Qualifizierung ist insbesondere prekär, da Haushaltshilfen überwiegend alleine arbeiten, mit komplexen Situationen insbesondere bei der Versorgung von Kindern und älteren, kranken Menschen konfrontiert sind und situativ Entscheidungen treffen müssen, die sich maßgeblich auf das Wohlergehen der Betreuten auswirken können. Systematische Fachkenntnisse aus der Altenpflege, der Pädagogik oder Hauswirtschaft könnten hilfreich sein, um Sachverhalte besser einzuschätzen. Darüber hinaus wäre professionelle Begleitung zielführend für die richtigen Entscheidungen in schwierigen Situationen. Für über 90 Prozent der Haushaltshilfen in Deutschland, die als Schwarzarbeiterinnen oder als Illegale tätig sind, erschließen sich solche Unterstützungsstrukturen nicht. Vielmehr ist die Lebenssituation von illegalen Haushaltshilfen oft noch zusätzlich davon bestimmt, dass sie in ständiger Angst vor Entdeckung leben.

\section{Warum arbeiten die meisten Haushaltshilfen in Deutschland unangemeldet?}

Dies ist vor allem eine Frage des Preises für die Dienstleistung. Während je nach Region der Stundenpreis für Schwarzarbeit im Durchschnitt zwischen acht und 12 Euro liegt, zahlen Haushalte für legale Angebote kommerzieller Art oder auch von Non-Profit-Anbietern im Schnitt zwischen 18 und 20 Euro oder mehr. Dieser große Unterschied in den Preisen ist insbesondere für die Haushalte älterer Menschen, die oft über geringe Budgets verfügen und gleichzeitig umfangreiche Dienstleistungen benötigen, das entscheidende Argument für informelle Beschäftigung von Haushaltshilfen. Aber auch die Haushaltshilfen selbst haben häufig ein großes Interesse an Schwarzarbeit, wenn sie zeitgleich noch andere Transferleistungen erhalten und einen Abzug durch das Erwerbseinkommen befürchten oder wenn sie im Rahmen des Ehegattensplittings für ein Familienzusatzeinkommen mit einem hohen Steuersatz belastet würden. Oft sind aber auch Gründe wie der bürokratische Aufwand für die Abwicklung Anlässe, die zu informeller Beschäftigung führen. Dies ist insbesondere dann der Fall, wenn nur punktuell Hilfen in geringem zeitlichem Umfang in Anspruch genommen werden.
Diese Ausgangssituation ist in Deutschland seit Langem bekannt und es sind mehrfach Anreize geschaffen worden, um den Anteil formaler Beschäftigung in Privathaushalten zu erhöhen. Allerdings greifen diese Anreize bisher nur in geringem Maße. Anhand von zwei Beispielen wollen wir darstellen, warum dies so ist. Die steuerliche Entlastung wird insbesondere von Seiten einschlägiger Politikbereiche gerne angeführt, wenn es um die Schaffung formaler Beschäftigung in Privathaushalten geht. Konkret bedeutet dies, dass Ausgaben für formal beschäftigte Haushaltshilfen steuerlich geltend gemacht werden können. Dieser Anreiz ist allerdings nur für jene Haushalte attraktiv, die tatsächlich auch Steuern zahlen. Im Falle der Haushalte von älteren Menschen, die nur selten Steuern zahlen, greift dieser Anreiz kaum. Gleichzeitig sind dies aber die Haushalte, die im Schnitt die größten fi-

\section{Der Mangel an Qualifizierung ist insbesondere prekär, da Haushaltshilfen mit komplexen Situationen, insbesondere bei der Versorgung von Kindern und älteren, kranken Menschen konfrontiert sind.}

nanziellen Belastungen für Haushaltshilfen haben. Als zweites Beispiel möchten wir die Minijobs anführen. Ihre Inanspruchnahme ist mit reduzierten Sozialabgaben und geringen Steuern verbunden. Durch den damit verbundenen Haushaltsscheck wird die Abwicklung deutlich erleichtert, weil die Minijobzentrale in diesen Fällen die Abführung von Steuern etc. übernimmt. Trotz dieser eindeutigen Vorteile wird der Minijob nur in wenigen Haushalten und auch nur in bestimmten Typen von Haushalten wahrgenommen. In den Genuss der subventionierten Sozialabgaben und des reduzierten Steuersatzes kommen vor allem Familien mit kleinen Kindern. Es sind jene Haushalte, die Haushaltshilfen nur in geringem Umfang benötigen und somit die Obergrenze von 400 Euro pro Monat auch nicht überschreiten. Andere Haushalte (und dies sind wiederum die Haushalte älterer Menschen) bleiben hier auch wieder weitestgehend außen vor, da sie vielfach umfassendere Hilfestellung benötigen, die die 400 Euro-Grenze deutlich überschreiten. Wie wir gerade aufgezeigt haben, stellen Steuererstattung und Minijob die zentralen Anreize für die Schaffung formaler Beschäftigungsverhältnisse in Privathaushalten dar. Sie greifen aber für die meisten Haushalte, insbesondere im Falle der Haushalte von älteren Menschen, nicht ausreichend. Mit dieser Ausrichtung der Instrumente kann der hohe Grad an informeller und 
damit auch häufig prekärer Beschäftigung in Deutschland vermutlich nicht nachhaltig reduziert werden.

\section{Wie hat es Frankreich geschafft, in großem Maße formelle Beschäftigung in Haushalten zu etablie- ren?}

Im Jahr 2005 hat der französische Sozialminister Jean-Luis Borloo eine Analyse zur Situation der Haushaltshilfen in Frankreich in Auftrag gegeben. Diese zeigte, dass Haushaltshilfen in hohem Maße informell beschäftigt waren, weil sich die meisten Haushalte formale Beschäftigung nicht leisten konnten. Darüber hinaus wurde deutlich, dass sich für viele Haushalte die Qualität und die Verlässlichkeit der Erbringung der Dienstleistung als wesentliches Problem darstellte, was unter anderem auf die fehlende Qualifizierung der Haushaltshilfen zurückgeführt wurde. Von dieser Analyse ausgehend wurde der sogenannte Borloo-Plan entwi-

\section{Über 500.000 neue sozialversicherungspflichtige Beschäftigungsverhältnisse wurden geschaffen. Illegale Beschäftigung konnte um 70 Prozent reduziert werden.}

ckelt. Dieser bestand im Kern aus einer Strategie, mit welcher bereits verfügbare Instrumente so spezifiziert wurden, dass diese beispielsweise tatsächlich für alle Haushaltstypen funktional wirksam werden konnten. Zudem wurden die Einzelinstrumente systematisch aufeinander bezogen, um Synergien erreichen zu können. Konkret bedeutet dies, dass gleichzeitig Instrumente zur Subvention von Sozialbeiträgen und zur Steuerreduktion, zur Förderung von Qualifizierung und zur Unternehmensentwicklung sowie zur Schaffung einer höheren Marktransparenz über die Einführung von Marken und lokalen Multiservicestrukturen implementiert wurden. Ein solch integrativer Ansatz ist aber erst möglich, wenn Ressortlogiken überwunden werden können. Dies gelang Jean-Luis Borloo, indem er alle relevanten politischen Ressorts und Stakeholder in den Entwicklungsprozess eingebunden und darüber hinaus Verantwortlichkeiten einzelner Akteur(inn)e(n) definiert hatte. Außerdem war seine Strategie prozessual angelegt. Dies bedeutet, dass die Wirkung der Instrumente kontinuierlich evaluiert wurde und auf diese Weise eine systematische Nachsteuerung erfolgen konnte. Verfahren zur Akkreditierung von Unternehmen und zur Zertifizierung von Beschäftigten wurden etabliert und flächendeckend genutzt. Es erfolgte die Entwicklung von neuen Weiterbildungen für Haushaltshilfen. Zur Verbesserung der Markttransparenz, insbesondere für Haushalte älterer Menschen, wurden Dachmarken etabliert, die jeweils ein breites Spektrum verschiedener Dienstleistungen zusammenfassen und qualitätsgesichert sind. Über eine systematische Verknüpfung von Subventionen, die fast alle bereits vor 2005 eingeführt worden waren, konnten die Preise so gestaltet werden, dass die Preislücke zur Schwarzarbeit deutlich reduziert wurde. Darüber hinaus gelang es, staatliche Hilfen wie beispielsweise Sozialhilfe zum Teil nicht mehr in Form von Geld auszuzahlen, sondern über Gutscheine, die nur zweckgebunden eingelöst werden konnten.

Die erste Phase des Borloo-Plans dauerte von 2005 bis 2009. Die in diesem Zeitraum erzielten Ergebnisse können sich sehen lassen. Über 500.000 neue sozialversicherungspflichtige Beschäftigungsverhältnisse von Haushaltshilfen wurden geschaffen. Illegale Beschäftigung konnte um 70 Prozent reduziert werden. Im Jahr 2009 wurde ein Umsatz von 19 Milliarden Euro in diesem Segment erwirtschaftet.

\section{Was kann Deutschland von Frankreich lernen?}

Die erfolgreiche Implementierung einer integrierten Strategie in Frankreich steht vermutlich in engem Zusammenhang mit der zentralistischen Struktur der Landes, die es erlaubt, top-down-Strategien durchzusetzen. Allein schon vor diesem Hintergrund kann die französische Strategie nicht direkt auf andere Länder, insbesondere wenn diese wie Deutschland eher föderalistisch geprägt sind, übertragen werden. Allerdings bietet das französische Beispiel trotzdem eine gute Orientierung dafür, wie erfolgreiche Strategien entwickelt werden können. Einen solchen Anhaltspunkt bilden die Instrumente, die in Frankreich zum Einsatz gekommen sind. Vergleicht man die französischen Instrumente mit den in Deutschland vorliegenden, so stellt sich ein überraschendes Ergebnis ein: Zur Mehrzahl der französischen Instrumente gibt es in Deutschland ein Pendant, seien es Steuerermäßigungen, reduzierte Sozialabgaben, Gründungskredite, Bildungsgutscheine, Haushaltsschecks u.a. Es bestehen jedoch große Unterschiede in der Ausgestaltung der jeweiligen Instrumente. Während die Spezifikation in Frankreich so ausgerichtet ist, dass die Instrumente tatsächlich eine hohe Funktionalität in diesem Segment entwickeln und Synergien durch deren kombinierten Einsatz erreicht werden können, greifen fast alle Instrumente in Deutschland nur unzureichend in diesem Segment. Dies wird beispielsweise daran deutlich, dass, wie oben gezeigt, nur einzelne Haushaltstypen daran partizi- 
pieren können, oder dass ein Instrument wie ein Mikrokredit zwar vorhanden ist, aber nicht an Gründer(innen) in diesem Segment vergeben wird, weil die Profitspanne im Schnitt geringer ist als in den Vergaberichtlinien vorgesehen. Neben der unzureichenden Spezifikation der Instrumente in Bezug auf die Erfordernisse in diesem Segment zeigt sich auch, dass die Instrumente nicht entlang der Prozesskette von der Erbringung bis zur Nutzung und Abwicklung der Dienstleistung systematisch aufeinander bezogen sind und damit keine Synergien generiert werden können. Als weiterer Unterschied zu Frankreich zeigt sich, dass die Instrumente in Deutschland in keiner homogenen oder abgestimmten Steuerungsstruktur verortet sind und damit die Wirkkraft implementierter Instrumente in der föderalen Komplexität schnell verpuffen kann. Als Fazit kann festgehalten werden, dass zwar notwendige Instrumente in Deutschland implementiert sind, diese jedoch nicht ihre Wirkung wie in Frankreich entfalten, sondern singulär und unkoordiniert wirken. Es erscheint dennoch plausibel, davon auszugehen, dass eine bessere Spezifikation der Instrumente auf das Segment familienunterstützende Dienstleistungen hin sowie deren systematische Verknüpfung, eingebettet in eine föderale Steuerungsstruktur, zu vergleichbaren Ergebnissen wie in Frankreich führen könnte. Solch eine integrierende Strategie könnte durchaus in einem Masterplan - vergleichbar dem französischen Borloo-Plan - abgebildet werden.

\section{Wie können nächste Schritte aussehen, um die unendliche Geschichte der informellen Beschäftigung in Deutschland endlich zu machen?}

Die deutsche Situation ist in den vergangenen zehn Jahren stark davon geprägt gewesen, dass hunderte von Modellprojekten, oft auf kommunaler Ebene, implementiert wurden, die mit externer Finanzierung versucht haben, qualitätsgesicherte und bedarfsgerechte Dienstleistungen von Haushaltshilfen anzubieten. ${ }^{3}$ Die Vielfalt der Ansätze zeigt ein großes Innovationspotential. Bisher gelingt es allerdings nicht, dieses auch nachhaltig zu nutzen, da sich die Projekte nach der Beendigung der externen Finanzierung kaum nachhaltig fortsetzen lassen, weil sie sich finanziell nicht selbst tragen können. Dies ist, wie oben schon festgestellt, der Situation geschuldet, dass die vorhandenen Instrumente nicht passfähig ausgerichtet sind.

Vor diesem Hintergrund wäre ein erster wichtiger Schritt die Durchführung einer Bestandsaufnahme der verfügbaren Instrumente, deren Wirkweisen und deren Inanspruchnahme. Unserer Kenntnis nach liegt bisher keine solche systematische Bestandsaufnahme vor. Auf der Basis einer Bestandsaufnahme könnte gezielt die passgenaue Spezifikation von Instrumenten, deren Kombinationsmöglichkeiten und auch deren Steuerung angegangen werden. Allerdings bedarf es dazu eines politischen Willens. Dies bedeutet, dass das Thema familienunterstützende Dienstleistungen dezidiert auf die politische Agenda von Bund, Ländern und Kommunen kommen muss. Dabei genügt es sicherlich nicht, wenn sich einzelne Ressorts mit Aktivitäten und Projekten zur Thematik befassen. Es ist vielmehr, um in der Richtung einer integrierten Strategie erfolgreich sein zu können, notwendig, ressortübergreifend tätig zu werden, indem beispielsweise interministerielle Arbeitsgruppen auf nationaler und auf Ebene der Bundesländer gebildet werden. Um solche Diskurse erfolgreich gestalten zu können, ist es, wie das französische Beispiel zeigt, wichtig alle relevanten Akteur(inn) e(n) aus Politik und Interessengruppen von Anfang an in die Prozesse einzubinden. ${ }^{4}$

Deutliche Anzeichen für einige der gerade skizzierten Schritte sind derzeit wahrnehmbar. Beispielsweise bereitet das Land Hessen eine Bundesratsinitiative zur Thematik vor. In verschiedenen Ministerien auf Bundesebene finden entsprechende Diskurse statt. Die einschlägige Wissenschaft begleitet einige dieser Prozesse. Dies sind derzeit noch zarte Pflänzchen, die es zu hegen und zu pflegen gilt; aber es ist ein Anfang in Deutschland gemacht.

Möglicherweise werden die deutschen Diskurse durch Aktivitäten der Europäischen Kommission und des Parlaments stimuliert. Dort ist das Thema bereits auf der politischen Agenda, wie einschlägige Veranstaltungen zeigen. ${ }^{5}$ Darüber hinaus wird im Sommer diesen Jahres eine Empfehlung der ILO (International Labour Organisation) herausgebracht werden, in welcher die arbeitsrechtlichen Bedingungen für den Arbeitsort Privathaushalt spezifiziert sind. ${ }^{6}$ Schließlich bleibt abzuwarten, ob der politische Diskurs in Deutschland nicht auch dadurch nochmals dynamisiert wird, wenn zum 1. Mai 2011 die vollständige Arbeitnehmer(innen)freiheit für Polen, Ungarn, Slowakei und andere wirksam wird. Dann wird es möglich sein, dass die nicht mehr illegalen Haushaltshilfen in andere Sektoren abwandern und dort legal arbeiten. Wie viele der derzeit 600.000 illegal in den Haushalten älterer Menschen beschäftigten Haushaltshilfen tatsächlich abwandern werden, ist nicht einzuschätzen. Vermutlich wird es jedoch einige Haushalte geben, die schnell einen Ersatz brauchen. Da die Politik hier nicht vorgesorgt hat, sind es voraussichtlich die Familien, insbesondere die Töchter, Schwiegertöchter und Ehefrauen, die schnell Lösungen finden müssen. Vielleicht verläuft dies ja nicht ganz „geräuschlos“ und das Thema kommt damit wieder an die Öffentlichkeit.

Weitere Informationen zum Thema: www.iwak-frankfurt. de/domesticservices.htm.

3 Das Projekt „Unternehmen Familie“, das im Auftrag des Bundesministeriums für Familie, Frauen, Senioren und Jugend und der BoschStiftung durchgeführt wurde, vermittelt einen guten Überblick über die heterogenen Modellprojekte. Vgl. www.bosch-stiftung.de.

4 Vgl. Dokumentation zur Veranstaltung „Wie kommt Deutschland zu einem Masterplan Haushaltsnahe Dienstleistungen?" am 6.12.2010 in der Hessischen Landesvertretung in Berlin, erhältlich im Internet: 〈www.iwak-frankfurt.de/domesticdeu.htm〉 (Zugriff: 8.4.11).

5 Vgl. Dokumentation der Konferenz "Successful strategies for creating declared employment in private homes" am 1.3.2011 in Brüssel, erhältlich im Internet: <www.iwak-frankfurt.de/domesticservices. htm> (Zugriff: 8.4.2011).

6 Vgl. Tomei, Manuela, Towards new international labour standards for domestic workers, Vortrag auf der o.g. Konferenz, erhältlich im Internet: <http://www.iwak-frankfurt.de/documents/ Brussels010311/brussels_tomei.pdf (Zugriff: 8.4.2011). 